\title{
A MaC Protocol With Dynamic Allocation Of Time Slots Based ON Traffic Priority IN WIRELESS BODY AREA NETWORKS
}

\author{
Sabin Bhandari and Sangman Moh \\ Department of Computer Engineering, Chosun University, Gwangju, South Korea
}

\begin{abstract}
In a wireless body area network (WBAN), wireless biomedical sensors are placed around, on, or inside the human body. Given specific requirements, WBANs can significantly improve healthcare, diagnostic monitoring, and other medical services. However, the existing standards such as IEEE 802.11 and IEEE 802.15.4 have some limitations to meet all the requirements of WBANs. Many medium access control $(M A C)$ protocols have been studied so far, most of which are derived from the IEEE 802.15.4 superframe structure with some improvements and adjustments. However, the MAC protocols do not provide the required quality of service $(Q o S)$ for various types of traffic in a WBAN. In this paper, a traffic-aware MAC (TA-MAC) protocol for WBANs is proposed, in which time slots are dynamically allocated on the basis of traffic priority, providing the required QoS. According to the performance evaluation results, the proposed TA-MAC is better than IEEE 802.15.4 MAC and the conventional priority-based MAC in terms of transmission time, system throughput, energy efficiency, and collision ratio.
\end{abstract}

\section{KEYWORDS}

Wireless body area network; Medium access control, Energy efficiency; Quality of service; Traffic priority; IEEE 802.15.4

\section{INTRODUCTION}

With the rapid advancement of electronic and wireless communication technologies, wireless sensor networks have grown significantly in a wide range of applications. A wireless body area network (WBAN) connects various medical sensors and appliances located inside and around the human body and is capable of monitoring health conditions remotely or within a hospital. The general architecture of a WBAN is depicted in Figure 1. A WBAN consists of biomedical sensors for monitoring physiological signals such as electromyography (EMG), electroencephalography (EEG), temperature, heart rate, and blood pressure [1]. Quality of service (QoS) is an important benchmark to achieve in WBANs. The key requirements in WBANs are small device size, low power consumption, negligible electromagnetic effects to the human body, short transmission delay, high reliability, and effective communication. The human body is a challenging environment to design an adaptable, dynamic, and flexible protocol for WBANs. WBANs have specific requirements and considerations that the IEEE 802.15.4 medium access control (MAC) protocol does not fully address [2]. 
MAC protocols play a vital role in prolonging the lifespan of a network by controlling the sources of energy waste such as packet collisions, overhearing, control packet overhead, and idle listening $[3,4]$. The main approaches adopted for energy savings in MAC protocols for WBANs are lowerpower listening (LPL), schedule contention, and time division multiple access (TDMA).

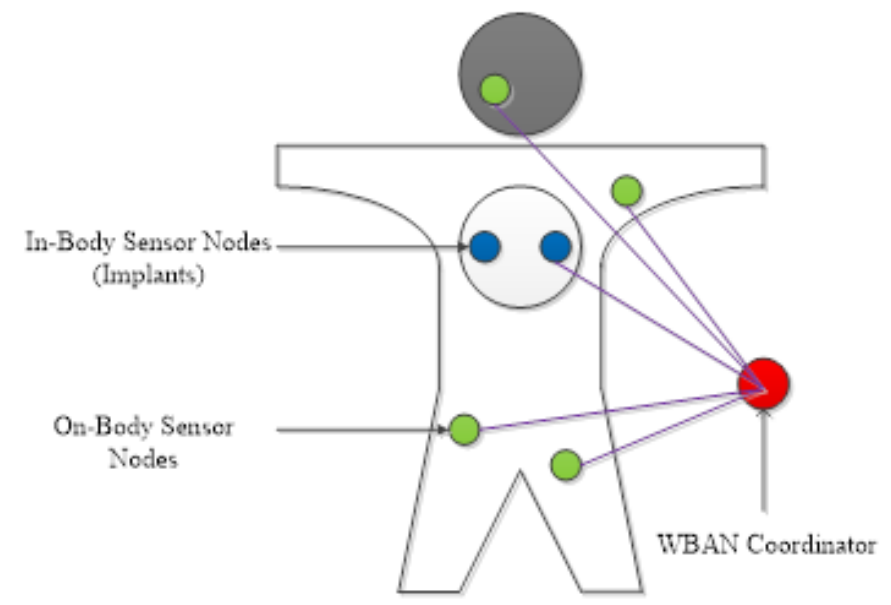

Figure 1. The general architecture of a WBAN

In the LPL mechanism, nodes wake up for a short duration to check the activity in the channel without receiving data. If the channel is busy, the node remains in an active state for receiving data, and other nodes go back to sleep mode. The LPL mechanism uses the non-persistent carrier sense multiple access (CSMA) protocol and preamble sampling technique to mitigate idle listening. Scheduled contention is a combination of scheduling and contention-based mechanism to avoid the problem of scalability and collision. In contention-based protocols, contending nodes attempt to access the channel for data transmission. Therefore, the probability of packet collision is significantly increased. In the carrier sense multiple access/collision avoidance (CSMA/CA) protocol, which is an example of contention-based MAC protocols, clear channel assessment (CCA) is performed by nodes before transmitting data. Even though these protocols are scalable, they cannot handle emergency situations in which the nodes with the same priority send critical data. TDMA MAC protocols divide the channel into multiple slots for data transmission. The protocols decrease idle listening and overhearing. However, the significant protocol overhead results in the protocols. Code division multiple access (CDMA) and frequency division multiple access (FDMA) schemes are also examples of scheduling mechanisms. However, CDMA and FDMA are not suitable for WBANs because of their high computational overhead and bandwidth limitation.

The IEEE 802.15.6 standard [5] describes physical (PHY) and MAC layers to address healthcare and nonmedical applications with diverse emerging requirements. The MAC layer in the IEEE 802.15.6 standard defines short-range wireless communication in and around the human body. In the standard, low complexity, low cost, ultra-low power, and highly reliable wireless communication are supported for use in close proximity to or inside the human body to support various entertainment and healthcare products and services.

The design of MAC protocols has a significant impact on energy efficiency, interference, reliability, and QoS provision. High channel utilization, error-free communications between nodes, low delay, and reduced energy consumption are key parameters for MAC protocols. One 
MAC protocol cannot satisfy all the requirements of various kinds of applications because the different kinds of protocols assume different hardware and applications [6].

The MAC protocol suitable for WBANs must cover up-to-date challenging issues with regard to WBAN topology and sensor constraints. In [7], the different approaches to PHY and MAC layer design for developing efficient mobile health applications are reviewed and extensively discussed. The key design issues, MAC layer parameters, energy consumption, coexistence, and channel modelling issues are investigated and summarized in $[8,9]$.

In this paper, a traffic-aware MAC protocol (TA-MAC) for WBANs is proposed, which prioritizes the sensor nodes by using a priority-aware CSMA/CA algorithm in the contention access period (CAP). For TA-MAC, we classify data traffic into four priorities and categorize the CAP into four sub-phases with dynamically changing length. This protocol is designed to support various QoS requirements for the data classified by priorities in WBANs. The proposed TA-MAC supports CAP and contention-free period (CFP). In the CAPs, the operation is based on a priorityguaranteed CSMA/CA procedure in which different WBAN nodes are assigned different priorities. The CFP is used to carry a number of data packets to the coordinator. The proposed TA-MAC operates on the basis of a beacon-enabled mode in which beacons are transmitted at the end of the superframe. In beacon-enabled mode, synchronization, association, and data transmission are controlled by the central coordinator by using periodic beacons. The beaconenabled mode uses a superframe that consists of active and inactive periods. The active period of the superframe consists of three parts: CAP using slotted CSMA/CA, CFP, and beacon as shown in Figure 2. The operation of the priority-guaranteed CSMA/CA is based on the IEEE 802.15.6 [5] and 802.11e [10] standards. The proposed TA-MAC considers a one-hop star topology network, and the entire operation is controlled by the WBAN coordinator.

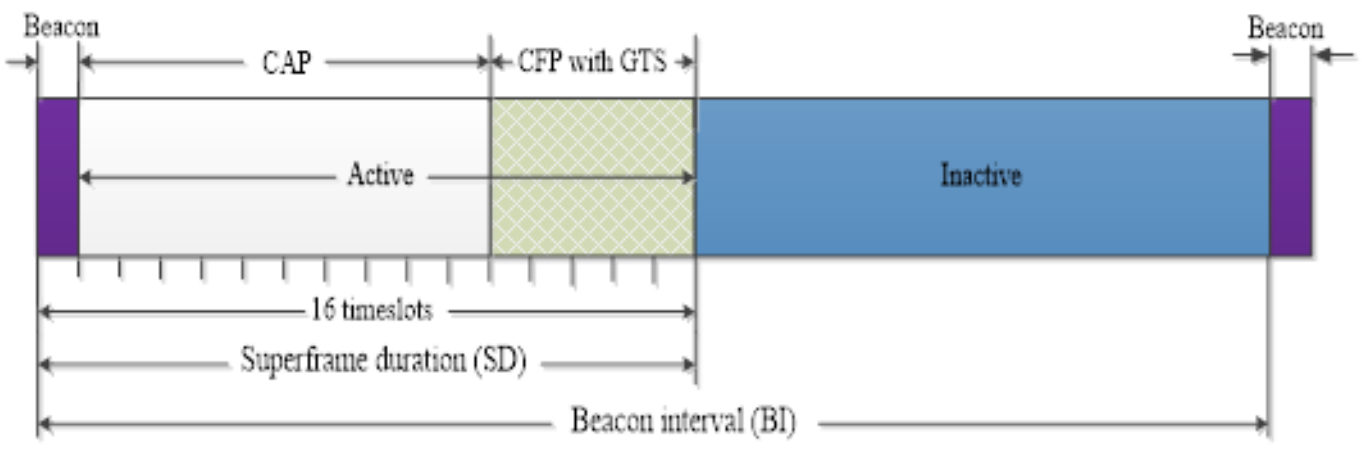

Figure 2. IEEE 802.15.4 superframe

The rest of this paper is organized as follows: In the following section, some related research is reviewed and discussed briefly. The proposed TA-MAC is presented in detail in Section 3. In Section 4, the performance of the proposed TA-MAC is evaluated via extensive computer simulation and then compared with IEEE 802.15.4 MAC and the conventional priority-based MAC protocol. Section 5 concludes the paper. 


\section{RELATED WORKS}

The IEEE 802.15.4 MAC protocol was designed for low-data-rate applications and is the most commonly used MAC protocol in wireless sensor networks to support low power and low data rate in cases where latency and bit rate are not critical [11]. The IEEE 802.15.4 MAC has the general characteristics of low power consumption, support for low-latency devices, star or peerto-peer operation, and dynamic device addressing.

The superframe structure of IEEE 802.15.4 MAC consists of a CAP, a CFP, and an inactive period. The CFP includes at most seven guaranteed timeslots (GTS). The superframe duration is denoted by the values of macBeaconOrder (BO) and macSuperFrameOrder (SO). The BO describes the beacon interval at which the coordinator may transmit its beacon. IEEE 802.15.4 MAC does not provide any prioritizing mechanism for the different kinds of data traffic, and lowpriority data can block the transmission of high-priority data.

There have been many significant developments of MAC protocols for WBANs. Most of the MAC protocols are already used for specific purposes, but they can be adopted with certain modifications to meet the requirements of WBANs. Most research has focused on the IEEE 802.15.4 standard for low-rate wireless personal area networks. An IEEE 802.15.3-based MAC protocol was designed as part of a body area networking system for multimedia applications [12]. A body MAC [13] was designed to provide energy-efficient and flexible operation in terms of bandwidth allocation and to support a sleep mode in WBANs. The MAC frame is adaptive and flexible for improving the efficiency of the sleep mode. The gateway controls the duration of the downlink, CAP, and CFP adaptively on the basis of traffic characteristics. The severe problem of the MAC protocol is that all nodes listen to the long preamble.

The context-aware MAC protocol [14] tries to support the real-time transmission of critical and emergency data. In order to address the problems of collision, idle listening, and overhearing, a TDMA-based scheme is used because the sensors transmit data only in their own slots. Data slots are assigned to sensors for contention-free transmission. One or more slots are assigned to sensor nodes for periodic or bursty applications according to their traffic characteristics. The contextaware MAC protocol performs well in terms of latency, but it does not respond to an emergency immediately.

In heartbeat-driven MAC protocol (H-MAC) [15], the TDMA approach boosts up the network efficiency by reducing idle listening and collisions. Although the H-MAC protocol reduces the extra energy cost for synchronization, it is not accessible to all sensors. In addition, the TDMA slots are not traffic adaptive.

In [16], multiple physical layers are taken into account including ultra-wideband. Also, the CAP uses mini slotted ALOHA scheme in order to enhancing the contention efficiency. The sufficient slot allocation in the CFP makes the protocol adaptive to different kinds of network traffic.

In [17], a multichannel management scheme for WBANs is presented. To reduce idle listening, the control channel is differentiated from data channels with different frequency bands. However, this scheme does not consider any priority differentiation mechanism. 
In the traffic priority and load-adaptive MAC protocol presented in [18], the transmission schedules of packets are determined based on their priorities. The superframe structure of the proposed protocol varies according to the traffic load and thereby minimizes energy consumption. In the traffic-adaptive MAC protocol (TaMAC) [19], a wake-up mechanism based on traffic and a wakeup radio to accommodate various traffic in a reliable manner are supported. In TaMAC, channels are tightly bounded by the superframe structure. The nodes for emergency traffic and the coordinator for on-demand traffic send wakeup radio signals to each other. TaMAC uses traffic information for low-power communication. It updates the traffic-based wake-up table but is inefficient for a dynamic topology.

In [20], a traffic-aware sensor MAC is introduced for collaborative body area sensor networks. The superframe structure dynamically varies based on the traffic load, and a multihop communication channel is used. However, it does not include the priority of different applications.

In [21], a traffic-aware dynamic MAC protocol (TAD-MAC) designed for WBANs is reported. Every node adapts its wakeup interval dynamically. The dynamic wakeup interval results in low energy consumption for idle listening, overhearing, collisions, and unnecessary wakeup beacon transmissions.

In [22], a priority-based channel access algorithm for contention-based MAC protocol (NPCAMAC) is designed to resolve the contention complexity issue. The algorithm categorizes the packets into four different priority levels by defining delay thresholds and divides the CAP into sub-phases. However, this algorithm may not consider the classification of continuous and discontinuous data or the use of GTSs in the CFP.

In [23], two CAPs are used in a hybrid and secure MAC protocol (PMAC) for WBANs for accommodating normal and critical data. On the other hand, one CFP is utilized to accommodate a number of data packets. Moreover, security keys are used for preventing unallowable access to WBANs.

In low-delay traffic-adaptive MAC protocol (LDTA- MAC) [24], GTSs are allocated dynamically based on traffic load to improve some of the shortcomings of the IEEE 802.15.4 MAC protocol. Data packets are transmitted in the current superframe after a successful GTS request. However, there is no consideration of traffic priority.

Existing standards such as IEEE 802.11 and IEEE 802.15.4 cannot meet all requirements of WBANs. Therefore, numerous MAC protocols have been studied. Many of them are the modification of the IEEE 802.15.4 superframe structure with some adjustments to address the requirements of WBANs. However, they do not support differentiated QoS for various kinds of traffic coexisting in a WBAN.

\section{Traffic-Aware Medium Access Control}

In this section, we present the proposed TA-MAC protocol in detail. The priority level of different kinds of data traffic, dynamic timeslot allocation algorithms, and data transfer procedures are discussed in the following subsections. 


\subsection{Traffic Priority}

Among WBAN applications, medical and consumer electronics (CE) signals represent the majority of data traffic in the network. Emergency traffic, which is directly related to the life of a patient (e.g., emergency alarm signals) should be regarded as the most important service and must be at the top priority level. Continuous medical traffic with common vital signals (e.g., EEG, electromyography) ranks in the second priority level. Discontinuous medical traffic (e.g., temperature, blood pressure) ranks in the third priority level. CE traffic (e.g., audio/videos transmitted in an event-driven manner) is ranked in the fourth priority level. The different levels of traffic priority are summarized in Table 1 .

Table 1. Levels of traffic priority.

\begin{tabular}{|l|c|l|}
\hline \multicolumn{1}{|c|}{ Traffic } & Priority & \multicolumn{1}{c|}{ Example } \\
\hline $\begin{array}{l}\text { Emergency } \\
\text { traffic (ET) }\end{array}$ & $P_{1}$ & Emergency alarm signal \\
\hline $\begin{array}{l}\text { On-demand } \\
\text { traffic (OT) }\end{array}$ & $P_{2}$ & $\begin{array}{l}\text { Continuous medical signal (e.g., } \\
\text { EEG, EMG) }\end{array}$ \\
\hline $\begin{array}{l}\text { Normal traffic } \\
\text { (NT) }\end{array}$ & $P_{3}$ & $\begin{array}{l}\text { Discontinuous medical signal (e.g., } \\
\text { temperature, blood pressure) }\end{array}$ \\
\hline $\begin{array}{l}\text { Nonmedical } \\
\text { traffic (NMT) }\end{array}$ & $P_{4}$ & Audio/video/data \\
\hline
\end{tabular}

\subsection{Dynamic Timeslot Allocation Algorithms}

The IEEE 802.15.4 MAC protocol comprises CAPs and CFPs. In this paper, we focus on the channel access of CAP because the performance of a CAP significantly influences the collision probability and the final throughput. When a number of nodes are densely deployed within a small area, contention complexity increases and leads to more energy consumption and collisions. Contention complexity is one of the requirements of WBANs that must be satisfied. Here we divide the CAP into sub-phases for each priority level of traffic; i.e., ET-CAP (Phase 1), ODTCAP (Phase 2), NT-CAP (Phase 3), and NMT-CAP (Phase 4) as shown in Figure 3. Nodes that transmit $P_{1}$ traffic can access channels through all phases from 1 to $4 . P_{2}$ can access channels from Phases 2 to 4 . Similarly, $P_{3}$ can access channels through Phases 3 and 4 . The node that transmits $P_{4}$ can use only Phase 4 to access the channel. Phase 1 always occupies the first time slot of the CAP [25]. To avoid wasted timeslot utilization, the length of sub-phases $L_{2}, L_{3}$, and $L_{4}$ dynamically change and are calculated by the coordinator according to a number of priority nodes on that sub-phase using equations (1), (2), and (3), respectively.

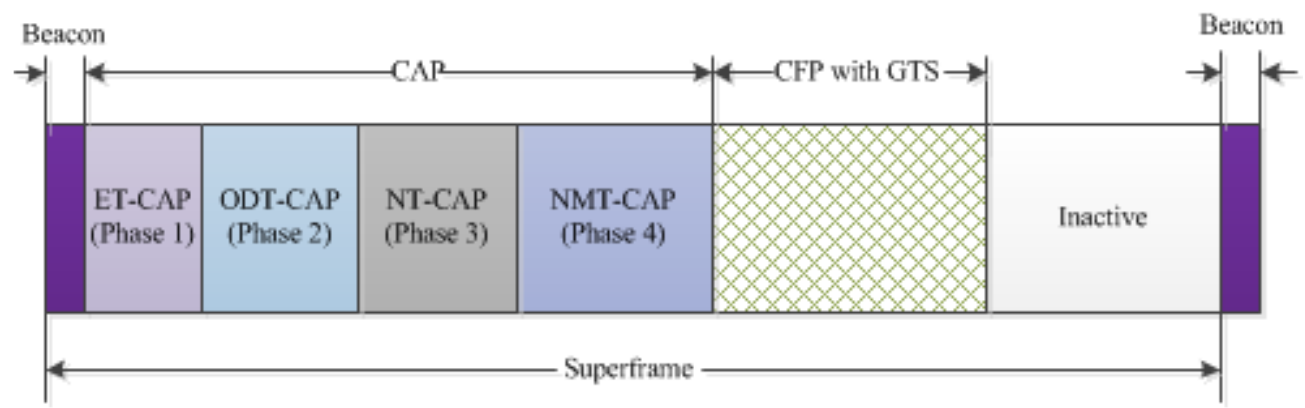

Figure 3. Superframe structure of the proposed MAC 
The length of Phase 1 in Figure 3 is fixed; it is one time slot long and always occupies the first time slot of the CAP. However, the lengths of the remaining phases are variable and represented as

$$
\begin{aligned}
& L_{2}=\frac{N_{2}(L-1)}{\sum_{i=2}^{4} N_{i}}, \\
& L_{3}=\frac{N_{3}(L-1)}{\sum_{i=2}^{4} N_{i}},
\end{aligned}
$$

and

$$
L_{4}=L-L_{3}-L_{2}-1
$$

where $N_{i}$ is the number of $i$-th priority nodes and $L$ is the CAP length in the unit of network timeslot.

The step-by-step algorithms for the slot allocation of the coordinator and the data transfer procedure for sensor nodes are shown in Figure 4 and Figure 5, respectively.

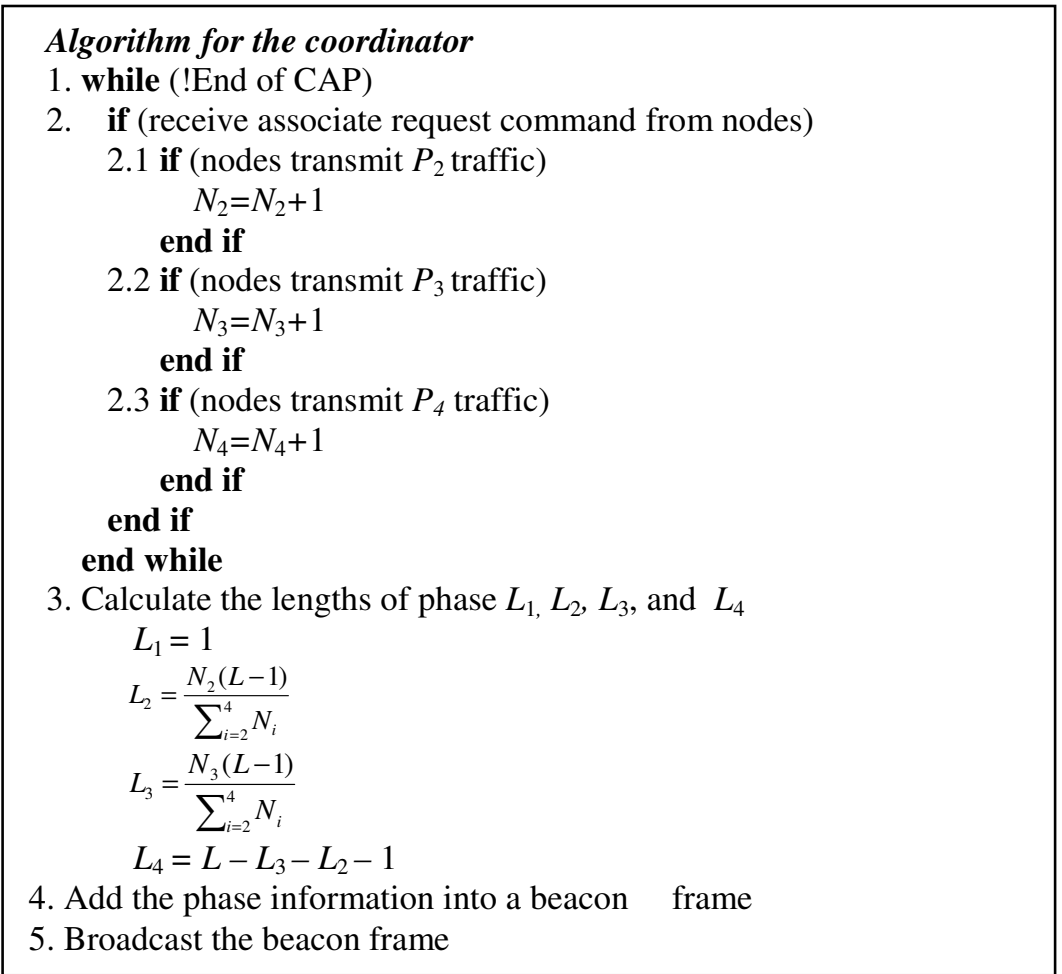

Figure 4. Algorithm for the coordinator

\subsection{Data Transmission Procedure}

In the IEEE 802.15.4 MAC protocol, the CAP is appropriate for the transmisison of short data and command messages, and the CFP is designed to transmit continuous data. The coordinator continuously broadcasts beacons to all nodes, and active nodes receive the beacons. The nodes 
send a request to the coordinator for the allocation of time slots in the CAP. According to the number of requests received, the coordinator also allocates TDMA slots in the CFP. To alleviate the collision of data traffic, a GTS scheduling criterion is defined. For P1 and P3, the data are transmitted immediately after successfully accessing the channel in the CAP. However, for P2 and P4, the nodes uniformly send GTS request command frames in the CAP to apply for GTS allocation. The data transfer procedures for different priorities of traffic are shown in Figures 6 and 7. In the CAP, TA-MAC employs the priority-based CSMA/CA procedure, which is based on the IEEE 802.15.6 standard. Each priority class has differentiated maximum/minimum contention windows and contention probability values to provide priority-based channel access to satisfy the QoS requirements of WBANs [5]

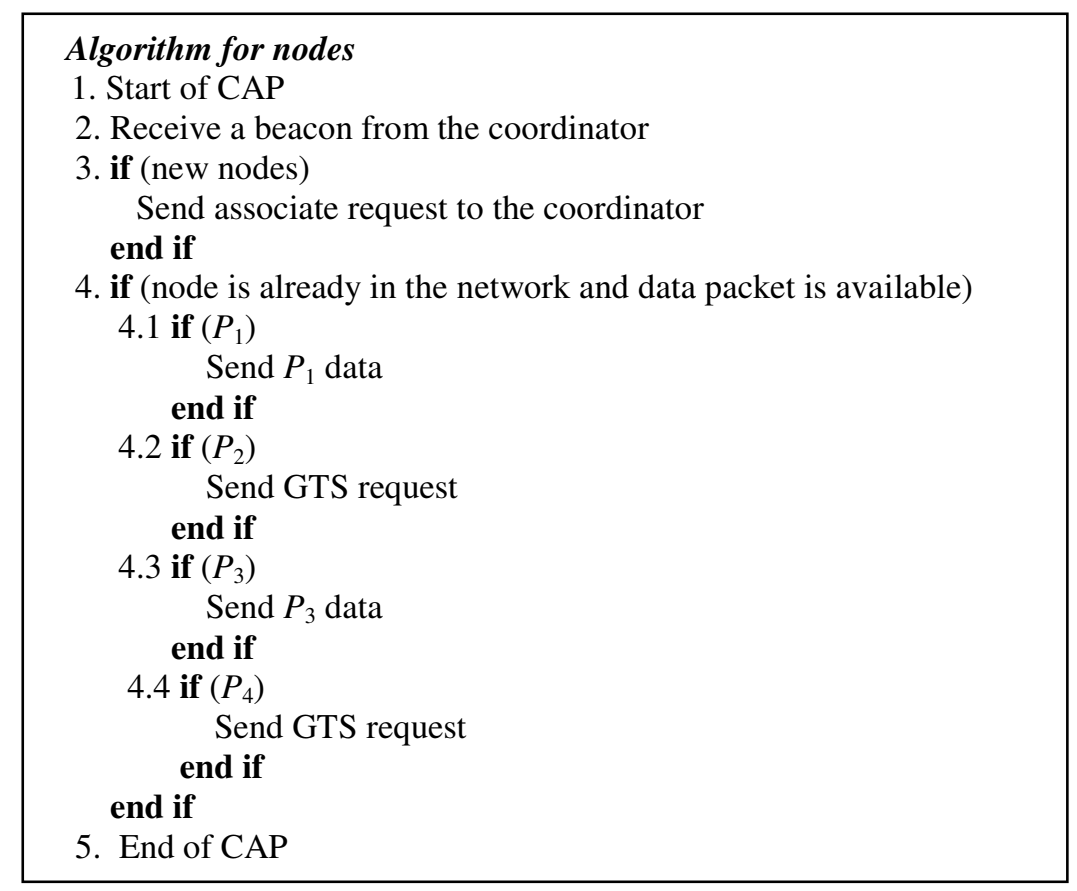

Figure 5. Algorithm for nodes

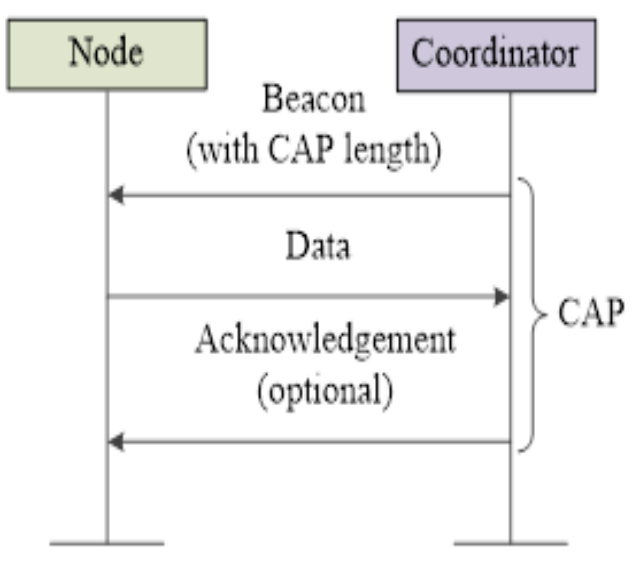

Figure 6. Data transfer for $P_{1}$ and $P_{3}$

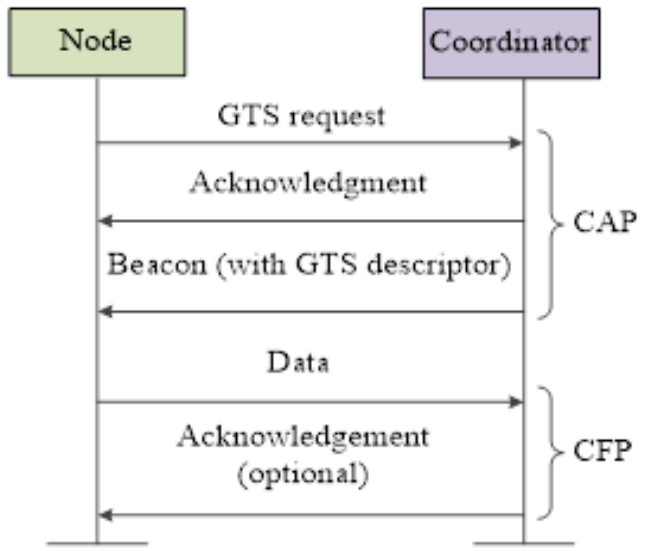

Figure 7. Data transfer for $P_{2}$ and $P_{4}$ 


\section{Performance Evaluation}

In this section, the performance of the proposed TA-MAC is evaluated via computer simulation and then compared to the existing IEEE 802.15.4 MAC [2] and NPCA-MAC [22] protocols.

\subsection{Performance Metrics}

The performance metrics used in our simulation are average transmission time, throughput, energy efficiency, and collision ratio. In this subsection, they are summarized in brief.

Average transmission time: In a WBAN, sensor nodes transmit their packets to the coordinator. In this paper, the transmission time is defined as the end-to-end delay from a sensor node to the coordinator. Many packets are transmitted from different sensor nodes to the coordinator for extended periods of time; therefore, the average transmission time is used in our performance study.

Throughput: Throughput refers to the actual level of network traffic put through the network across an end-to-end communication path between a transmitting node and one or more receiving nodes. It is defined as the average transmission rate of successfully delivered bits per second over a communication channel.

Energy Efficiency: Energy efficiency is one of the key requirements for WBAN MAC protocol designs. Energy consumption depends on the behaviour of the nodes on the network. A network with heavy traffic has higher energy consumption than one with low traffic activity. To comprehensively compare the MAC protocols, we calculated average energy consumption per bit to evaluate energy efficiency. The energy consumption per bit is defined as the total energy consumption over the total number of bits delivered during a simulation run [26].

Collision Ratio: The main objectives for WBANs are to achieve the maximum throughput, minimum delay, and maximum lifespan by controlling the primary sources of energy waste; i.e., idle listening, overhearing, control packet overhead, and collision. A collision occurs when two or more nodes transmit data packets simultaneously. If the network contains a large number of nodes, the number of collisions increases. In general, the network load can be indicated as the ratio of the number of collisions over the number of packets transmitted.

\subsection{Simulation Environment}

Our performance simulation was extensively performed by the ns- 2 network simulator version 2.35. It is assumed that several biomedical sensors are attached to or implanted into the human body. The sensors collect the sensed data and transmit them to the central coordinator, resulting in a star topology. All sensor nodes are randomly deployed within a $5 \mathrm{~m}$ radius around the central coordinator, and data are transmitted using one-hop communication [22]. 
Table 2. Simulation parameters.

\begin{tabular}{|l|c|}
\hline \multicolumn{1}{|c|}{ Parameter } & Value \\
\hline Channel rate & $250 \mathrm{kbps}$ \\
\hline Frequency band & $2.4 \mathrm{GHz}$ \\
\hline Symbol times & $16 \mu \mathrm{s}$ \\
\hline Superframe duration & $122.88 \mathrm{~ms}$ \\
\hline Transition time & $194 \mu \mathrm{s}$ \\
\hline aUnitBackoffPriod & $20 \mathrm{symbols}$ \\
\hline macBeaconOrder (BO) & $3^{*}$ \\
\hline macMaxCSMABackoffs & $5^{*}$ \\
\hline macMinBE & $3^{*}$ \\
\hline macMaxBE & $5^{*}$ \\
\hline Idle power & $712 \mu \mathrm{W}$ \\
\hline Transmission power & $36.5 \mathrm{~mW}$ \\
\hline Reception power & $41.4 \mathrm{~mW}$ \\
\hline
\end{tabular}

* No specific unit is used for the four system parameters [2].

The network parameters used for simulation are summarized in Table 2 as in [27]. Only 20 percent of the nodes generate $\mathrm{P}_{1}$ traffic during each simulation. In our simulation, the physical layer parameters conform to the IEEE 802.15.4 standard. The protocols under evaluation are based on the beacon enable mode, and the values of the two parameters $\mathrm{SO}$ and $\mathrm{BO}$ in Table 2 determine the superframe duration (SD) and the beacon interval (BI), respectively. We assume that the channel gain is stationary for both control and data packet transmission durations. Packet loss is only due to collision, and small-scale fading is neglected. Poisson arrival is assumed to model the random packet arrival process [26]. In addition, we consider only the general application scenario of WBANs where all data transmissions are initiated by sensor nodes toward the coordinator. In the proposed TA-MAC, the contention window values based on priority are modelled as in the IEEE 802.15.6 MAC standard. This is because not only random backoff exponential but also contention window values are not prioritized in the IEEE standard.

\subsection{Simulation Results and Discussion}

Figure 8 shows the average transmission time as a function of the number of nodes. IEEE 802.15.4 MAC operates slotted CSMA/CA without a priority-based channel access policy whereas TA-MAC and NPCA-MAC perform slotted CSMA/CA with a priority-based channel access policy. Thus, as shown in Figure 8, the average transmission time of IEEE 802.15.4 MAC has the largest delay compared to the proposed TA-MAC and the conventional NPCA-MAC. Also, the average transmission time is gradually increased as the number of nodes increases. 
International Journal of Computer Networks \& Communications (IJCNC) Vol.11, No.4, July 2019

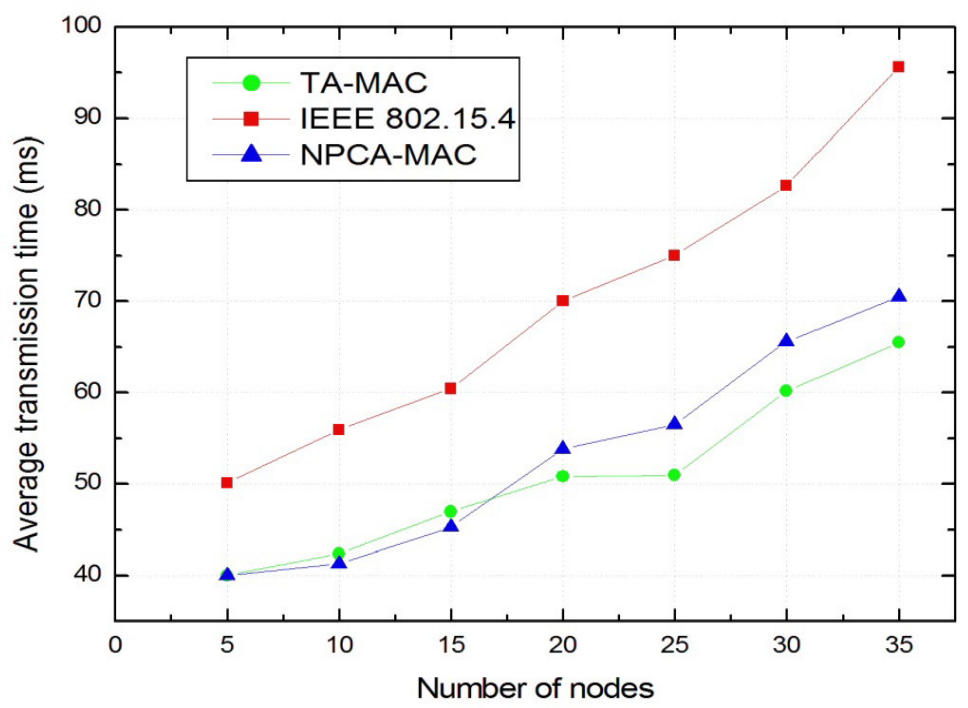

Figure 8. Average transmission time

Figure 9 shows the overall network throughput of TA-MAC, IEEE 802.15.4 MAC, and NPCAMAC. The network throughput is the total amount of data packets received by the central coordinator in a time interval. Here, we can see that the throughput of all three protocols increases as the number of sensor nodes increases. When the network has low traffic load (i.e., less than 15 sensor nodes), all the three protocols perform similarly. With the increased number of sensor nodes, TA-MAC shows improved throughput over NPCA-MAC and IEEE 802.15.4 MAC. In fact, the classification of data transfers and the allocation of GTSs makes TA-MAC outperform NPCA-MAC and IEEE 802.15.4 MAC.

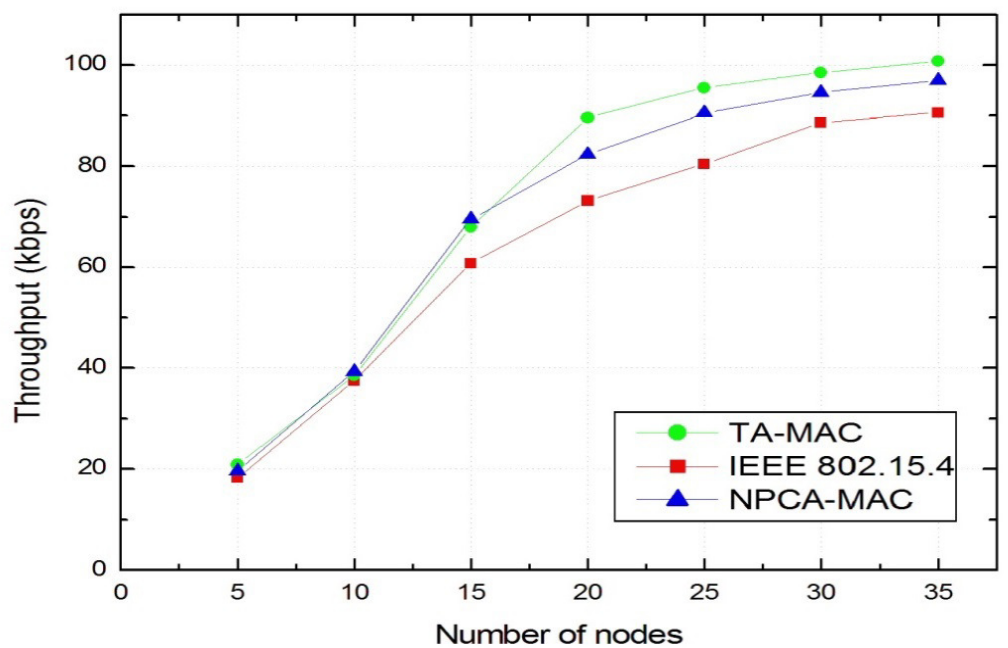

Figure 9. Network throughput 


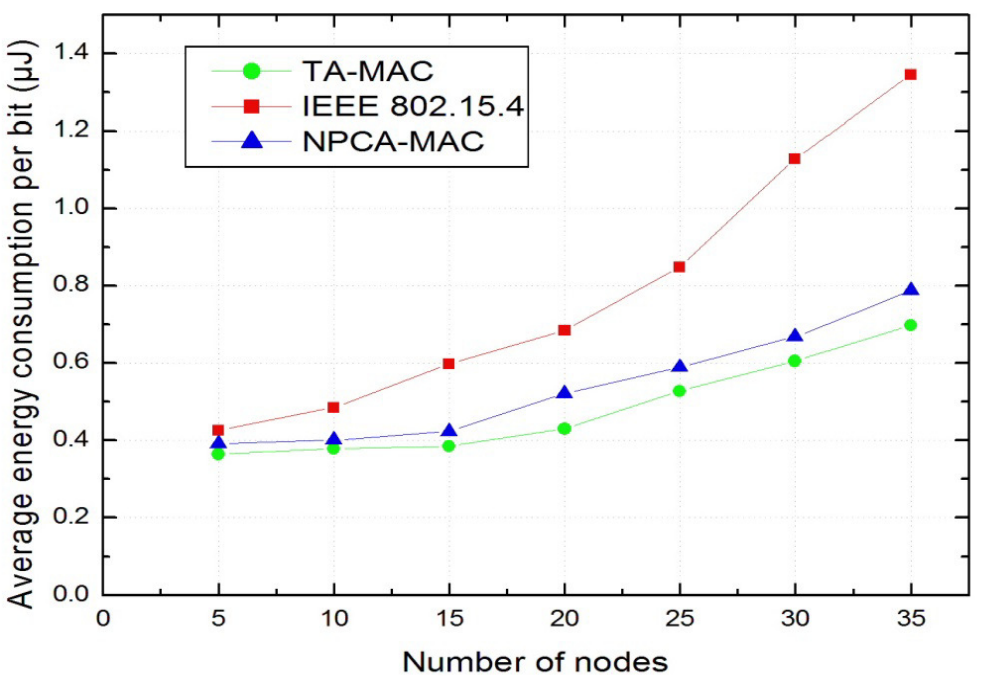

Figure 10. Average energy consumption per bit

The average energy consumption per bit is illustrated in Figure 10. The proposed TA-MAC and NPCA-MAC show better performance than IEEE 802.15.4 MAC in all network scenarios. However, TA-MAC shows slightly better performance than NPCA-MAC when the number of nodes is greater than 15. In general, packet collision and retransmission result in more energy consumption. As the number of nodes increases, the energy consumption of IEEE 802.15.4 MAC is increased because of increased contention complexity. However, in the proposed TA-MAC, prioritized channel access with differentiated contention window, classification of data transfer, and backoff exponential values reduce the contention complexity, number of collisions, and packet retransmissions.

The collision ratio is shown in Figure 11. The number of collisions increases proportionately with the number of nodes in the network. IEEE 802.15.4 MAC shows a large number of collisions compared to TA-MAC and NPCA-MAC. In IEEE 802.15.4 MAC, the collision ratio increases when the number of nodes is greater than 15. This is because the slotted CSMA/CA without prioritization does not resolve the contention complexity issue. However, the number of collisions for TA-MAC and NPCA-MAC is lower than IEEE 802.15.4 MAC due to the prioritized CCA and random backoff algorithm. For the classification of continuous and discontinuous data transfer, the proposed TA-MAC shows marginally better performance than NPCA-MAC as the number of nodes is increased. 


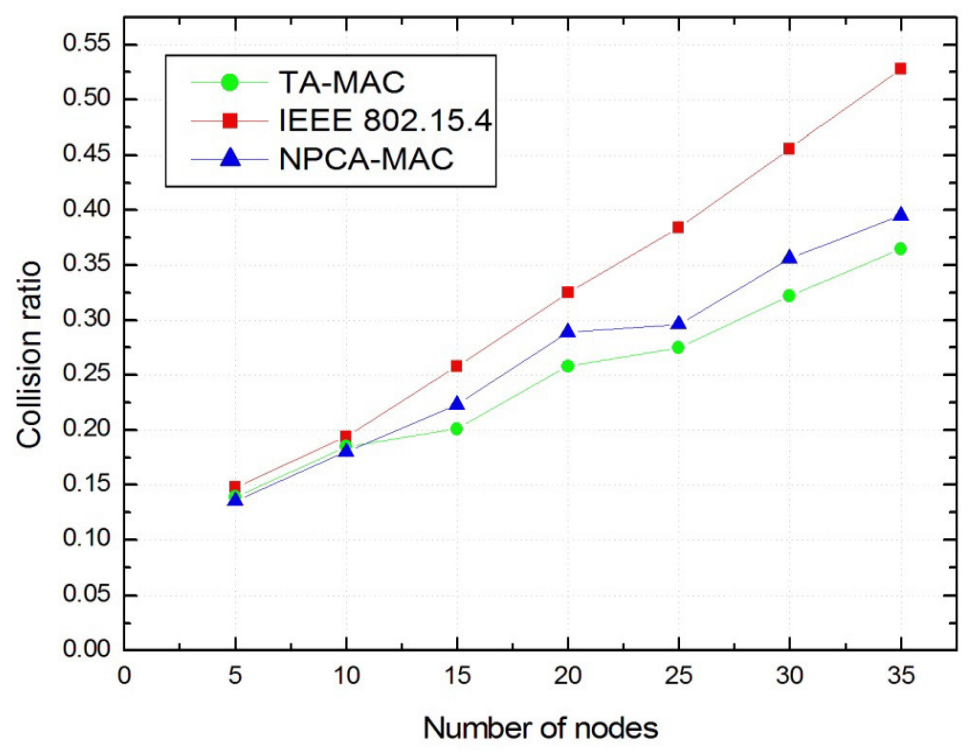

Figure 11. Collision ratio

Figure 12 shows the effects of four different priority levels in terms of average transmission time. In Figure 12, the average transmission time of each priority traffic is shown for TA-MAC and IEEE 802.15.4 MAC, but the average transmission time of NPCA-MAC is not shown because it is almost the same as that of the proposed TA-MAC. This is primarily because NPCA-MAC also divides the CAP into four sub-phases according to the different priority levels of traffic as in TAMAC. In NPCA-MAC, however, the continuous and discontinuous data transfer and the use of GTSs were not taken into account. From Figure 12, we can infer that there is no difference in the transmission time among all priority traffic under the same MAC. However, TA-MAC shows better performance than IEEE 802.15.4 MAC.

In the IEEE 802.15.4 based MAC, the collision ratio increases sharply as the number of nodes and the packet arrival rate are increased. The increased collision ratio causes a serious waste of radio and network resources. The emergency nodes transmit a small-size data packet in a small time interval. The throughput is not necessarily a major concern for such an emergency medical traffic. For CE applications, however, high data rate is a must for improved user experience. Therefore, the throughput is a key performance metric for CE applications and needs to be given prime importance. In the proposed TA-MAC, the GTS slots are assigned to the continuous medical data traffic and CE traffic. Because the number of GTS slots is limited, a significant number of collisions are more likely to occur and thereby degrade the system performance with regard to delay and energy efficiency. 


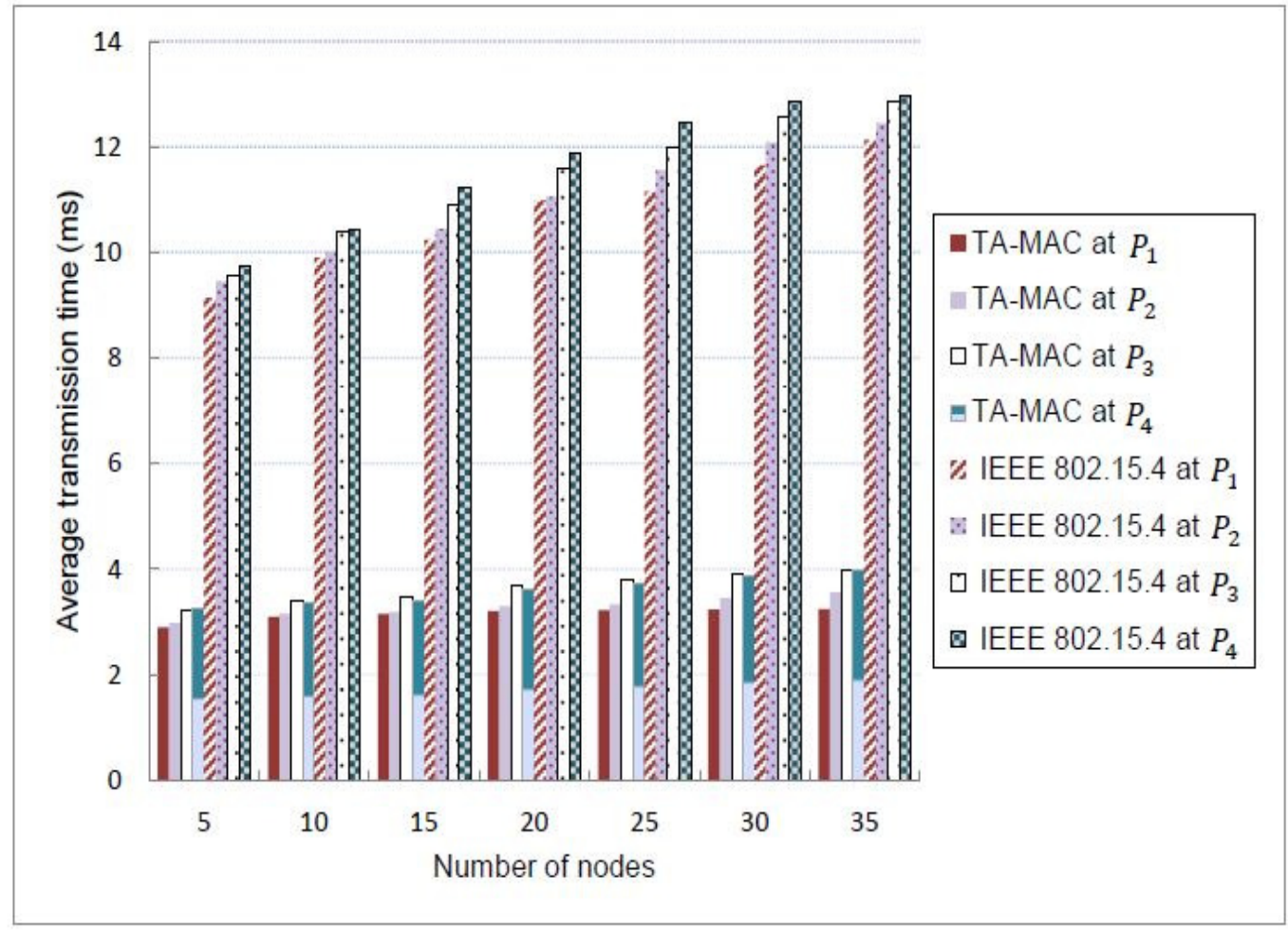

Figure 12. Effects of different priority levels

\section{Conclusions}

In this paper, we have proposed a novel traffic-aware MAC named TA-MAC in order to support various QoS requirements in WBANs. The proposed TA-MAC differentiates the access phase of the CAP and classifies the transfer procedure of priority-based traffic in WBANs. TA-MAC uses CFPs for continuous and large amounts of data. According to the simulation results, TA-MAC showed substantial improvements in terms of transmission time, throughput, energy efficiency, and collision ratio compared to IEEE 802.15.4 MAC and NPCA-MAC.

The various kinds of wireless network services based on IEEE 802.11 (Wi-Fi), IEEE 802.15.1 (Bluetooth), and IEEE 802.15.4 (ZigBee) are possible nowadays in the industrial, scientific, and medical (ISM) band. The technologies coexist in the same frequency band and, thus, may cause mutual interference. WBANs operating in highly coexisting environments may suffer from long packet delay, low network throughput, and high-energy consumption. A possible future work is to apply cognitive radio and multichannel access to the design of a MAC protocol for WBANs in order to mitigate the coexisting interference and improve network performance including QoS.

\section{ACKNOWLEDGEMENTS}

A preliminary version of this work was presented at the InfoWare 2017 Conference, Nice, France, July 2017 [28]. The authors wish to thank the editor and anonymous referees for their helpful comments on improving this paper. This research was supported in part by research fund from Chosun University, 2018. Correspondence should be addressed to Dr. Sangman Moh (smmoh@chosun.ac.kr). 


\section{REFERENCES}

[1] D. Fernandes, A. G. Ferreira, R. Abrishambaf, J. Mendes, andJ. Cabral, (2018) "Survey and taxonomy of transmissions power control mechanisms for wireless body area networks," IEEE Communications Surveys and Tutorials, vol. 20, no. 2, pp. 1292-1328

[2] IEEE, (2006) "IEEE Std.802.15.4: Wireless medium access control (MAC) and physical layer (PHY) specifications for low data rate wireless personal area networks (WPAN)," Piscataway, NJ, USA

[3] X. Yang, L. Wang, and Z. Zhang, (2018) "Wireless body area networks MAC protocol for energy efficiency and extending lifetime," IEEE Sensors Letters, vol. 2, no. 1, pp. 1-4

[4] B. Liu, Z. Yan, and C. W. Chen, (2017) "Medium access control for wireless body area networks with QoS provisioning and energy efficient design," IEEE Transactions on Mobile Computing, vol. 16, no. 2, pp. 422-434

[5] S. Ullah, M. Chen, and K. Kwak, (2012) "Throughput and delay analysis of IEEE 802.15.6 based CSMA/CA protocol," Journal of Medical Systems, vol. 36, no. 6, pp. 3875-3891

[6] S. Bhandari and S. Moh, (2015) "A survey of MAC Protocols for cognitive radio body area networks," Sensors, vol. 15, pp. 9189-9209

[7] E. Kartsakli, A. Lalos, A. Antonopoulos, S. Tennina, M. Renzo, L. Alonso, and C. Verikoukis, (2014) "A survey on M2M systems for mHealth: A wireless communications perspective," Sensors, vol. 14, pp. 18009-18052

[8] R. Cavallari, F. Martelli, R. Rosini, C. Buratti, and R. Verdone, (2014) "A survey on wireless body area networks: Technologies and design challenges," IEEE Communications Surveys \& Tutorials, vol. 16, pp. 1635-1657

[9] T. Le and S. Moh, (2015) "Interference mitigation schemes for wireless body area sensor networks: A comparative survey," Sensors, vol. 15, pp. 13805-18838

[10] S. Mangold, S. Choi, G. R. Hiertz, O. Klein, and B. Walke, (2003) "Analysis of IEEE 802.11e for QoS support in wireless LANs,” IEEE Wireless Communications, vol. 10, No. 3, pp. 40-50

[11] N. F. Timmons and W. G. Scanlon, (2004) "Analysis of the performance of IEEE 802.15.4 for medical sensor body area networking," in Proc. of 1st Annual IEEE Communications Society Conference on Sensor and Ad Hoc Communications and Networks, pp. 16-24

[12] T. Falck, J. Espina, J. P. Ebert, and D. Dietterle, (2006) "BASUMA - The sixth sense for chronically ill patients," in Proc. of International Workshop on Wearable and Implantable Body Sensor Networks, pp. 1-6

[13] G. Fang and E. Dutkiewicz, (2009) "BodyMAC: Energy efficient TDMA-based MAC protocol for Wireless Body Area Network," in Proc. of 9th International Symposium on Communications and Information Technology, pp. 1455-1459

[14] Z. Yan and B. Liu, (2011) "A context aware MAC protocol for medical wireless body area network," in Proc. of 7th Int. Wireless Communication and Mobile Computing Conf. (IWCMC 2011), pp. 21332138

[15] L. Huaming and T. Jindong, (2010) "Heartbeat-driven medium-access control for body sensor networks," IEEE Transactions on Information Technology in Biomedicine, vol. 14, no. 1, pp. 44-51 
International Journal of Computer Networks \& Communications (IJCNC) Vol.11, No.4, July 2019

[16] C. Li, L. Wang, J. Li, B. Zhen, H.-B. Li, and R. Kohno, (2009) "Scalable and robust medium access control protocol in wireless body area networks," in Proc. of IEEE 20th International Symposium on Personal, Indoor and Mobile Radio Communications, pp. 2127-2131

[17] W. Lee, S. H. Rhee, Y. Kim, and H. Lee, (2009) “An efficient multi-channel management protocol for wireless body area networks," in Proc. of International Conference on Information Networking, pp.1-5

[18] I. Anjum, N. Alam, M. A. Razzaque, M. Mehedi Hassan, and A. Alamri, (2013) "Traffic priority and load adaptive mac protocol for qos provisioning in body sensor networks," International Journal of Distributed Sensor Networks, vol. 2013, pp. 1-9

[19] K. S. Kwak and S. Ullah, (2010) "A traffic-adaptive MAC protocol for WBAN," in Proc. of IEEE GLOBECOM Workshops, pp. 1286-1289

[20] O. Md. Rahman, C. S. Hong, S. Lee, and Y.-C. Bang, (2011) "ATLAS: A traffic load aware sensor MAC design for collaborative body area sensor networks," Sensors, vol. 11, no.12, pp. 11560-11580

[21] M. M. Alam, O. Berder, D. Menard, and O. Sentieys, (2012) "TAD-MAC: traffic-aware dynamic MAC protocol for wireless body area sensor networks," IEEE Journal on Emerging and Selected Topics in Circuits and Systems, vol. 2, no. 1, pp. 109-119

[22] B. Kim and J. Cho, (2012) "A novel priority-based channel access algorithm for contention-based MAC protocol in WBANs," in Proc. of 6th International Conference on Ubiquitous Information Management and Communication (ICUIMC 2012), pp. 1-5

[23] S. Ullah, M. Imran, and M. Alnuem, (2014) "A hybrid and secure priority-guaranteed MAC protocol for wireless body area network," International Journal of Distributed Sensor Networks, vol. 2014, pp. $1-7$

[24] C. Li, B. Hao, K. Zhang, Y. Liu, and J. Li, (2011) "A novel medium access control protocol with low delay and traffic adaptivity for wireless body area networks," Journal of Medical Systems, pp. 12651275

[25] S. Jin, Z. Weixia, and Z. Zheng, (2013) "Priority-based adaptive timeslot allocation scheme for wireless body area network," in Proc. of 13th International Symposium on Communications and Information Technologies, pp. 609-614

[26] Y. Zhang and G. Dolmans, (2010) "Priority-guaranteed MAC protocol for emerging wireless body area networks," Annals of Telecommunications, vol. 66, pp. 229-241

[27] X. Liang and I. Balasingham, (2007) "Performance analysis of the IEEE 802.15.4 based ECG monitoring network," in Proc. of 7th International Conferences on Wireless and Optical Communications, pp. 99-104

[28] S. Bhandari, K. Singh, and S. Moh, (2017) "Traffic-Aware Medium Access Control Protocol for Wireless Body Area Networks," in Proc. of InfoWare 2017 Conference, pp. 1-6 


\section{AuTHORS}

Sabin Bhandari received his B.E. degree in electronics and communication engineering from Tribhuvan University, Nepal in 2010. He worked as a lecturer and project assistant in Kantipur Engineering College, Nepal from 2011 to 2014. He completed his M.E. degree in computer engineering from Chosun University, Gwangju, South Korea in 2016. His current research interests include wireless body area networks, wireless sensor networks, and cognitive radio.

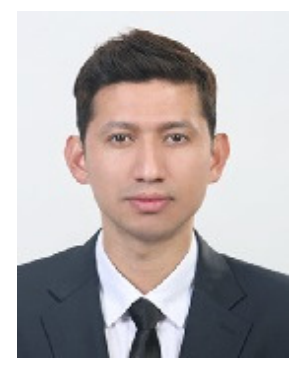

Sangman Moh received his $\mathrm{Ph} . \mathrm{D}$. degree in computer engineering from Korea Advanced Institute of Science and Technology (KAIST), Korea in 2002. Since late 2002, he has been a professor at the Dept. of Computer Engineering at Chosun University, Korea. From 2006 to 2007, he was on leave at Cleveland State University, USA. His research interests include mobile computing and networking, ad hoc and sensor networks, cognitive radio networks, and parallel and distributed computing systems. He has published more than 200 papers in international and domestic journals and conference proceedings, and has held more than 40 overseas and domestic patents. He serves on the program committees of international conferences and workshops in his areas of interest as a chair or member. Dr. Moh is a member of the IEEE, the ACM, the IEICE, the KIISE, the IEIE, the KIPS, the KICS, the KMMS, the IEMEK, the KISM, and the KPEA.

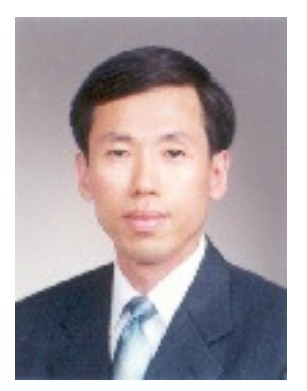

